\title{
Biliary self-expandable metallic stent using single balloon enteroscopy assisted ERCP - overcoming limitations of current accessories
}

\author{
Rolando Pinho ${ }^{1}$, Luisa Proença ${ }^{1}$, Luis Alberto ${ }^{1}$, João Carvalho ${ }^{1}$, Teresa Pinto-Pais ${ }^{1}$, Carlos Fernandes ${ }^{1}$, \\ Iolanda Ribeiro ${ }^{1}$, Tiago Pereira ${ }^{2}$ and José Fraga ${ }^{1}$
}

Departments of ${ }^{1}$ Gastroenterology and ${ }^{2}$ Radiology. Centro Hospitalar de Vila Nova de Gaia. Portugal

\begin{abstract}
Device assisted enteroscopy (DAE) techniques have been recently developed for the diagnosis and treatment of small bowel diseases. These techniques can also be used to perform endoscopic retrograde cholangiopancreatography $(\mathrm{ERCP})$ in patients with surgically altered anatomies. The main difficulties with DAE-ERCP are related to type of surgery, to the different dimensions and lack of frontal view of the enteroscope and to the resulting limitations with the use of standard accessories, resulting in the need of dedicated accessory devices. Although most ERCP techniques have been successfully performed with DAE-ERCP, biliary self-expandable metallic stents (SEMS) have not yet been used, as dedicated biliary SEMS for the enteroscope are lacking. The authors present a case report showing a new method to place standard biliary trough-thescope SEMS with DAE-ERCP, using a different technique of stent deployment.
\end{abstract}

Key words: Enteroscopy. Endoscopic retrograde cholangiopancreatography. Self-expandable metallic stent. Self-expandable metallic stents.

\section{INTRODUCTION}

Device assisted enteroscopy (DAE) is the standard technique to explore the small bowel. Available systems include double balloon (DBE), single balloon (SBE) and spiral-enteroscopy (SE). With the help of dedicated devices -an overtube with either a balloon attached at the distal tip or a spiral along the overtube- deep small bowel exploration is now possible (1).

These techniques have also been used to access surgically excluded intestinal segments, such as those resulting

\footnotetext{
Received: 02-04-2013
}

Accepted: 24-05-2013

Correspondence: Rolando Taveira Pinho. Department of Gastroenterology. Centro Hospitalar de Vila Nova de Gaia. Portugal

e-mail: rolandopinho@gmail.com from partial gastrectomy or total gastrectomy with Y-Roux reconstruction, partial gastrectomy with long jejunal afferent limbs or gastric bypass surgery for morbid obesity.

Access to the pancreatic and biliary ducts in these patients is also possible with all available DAE techniques (2-5). The greatest limitations using DAE to perform endoscopic retrograde cholangiopancreatography (ERCP) are the frontal view of the enteroscope (as opposed to the lateral view of conventional duodenoscopes), the smaller caliber channel of $2.8 \mathrm{~mm}$ and the longer channel length of $200 \mathrm{~cm}$ (6). These limitations turn the procedure challenging and more demanding than standard ERCP. Moreover, available standard ERCP accessories cannot be used with the enteroscope, due to either short length or large caliber. Specially adapted, usually longer, accessories are commercially available, although with limited variety and local availability. There are no metallic biliary self-expandable stents adapted to use within a $2.8 \mathrm{~mm}$ large and $200 \mathrm{~cm}$ long channel, turning the standard "through the scope" technique of stent deployment impossible in these cases (3).

A new technique for biliary SEMS deployment in DAE-ERCP is described.

\section{CASE REPORT}

The case reports to an 80 years old female patient with past history of partial gastrectomy with Y-Roux reconstruction for gastric adenocarcinoma (pT1 N0 M0) 3 years ago. She presents with jaundice and itching from gastric cancer recurrence causing biliary stenosis.

Pinho R, Proença L, Alberto L, Carvalho J, Pinto-Pais T, Fernandes C, Ribeiro I, Pereira T, Fraga J. Biliary self-expandable metallic stent using single balloon enteroscopy assisted ERCP - overcoming limitations of current accessories. Rev Esp Enferm Dig 2013;105:561-564. 
The physical examination was unremarkable except for scleral and cutaneous jaundice.

Laboratory test results revealed normal leucocyte count, with elevated total bilirubin $(9.16 \mathrm{mg} / \mathrm{dL}$ [0.1-1.1]), conjugated bilirubin $(8.00 \mathrm{mg} / \mathrm{dL}$ [0.1-0.3]), AST (104 U/L [4-27]), ALT (205 U/L [4-34]), gamma-glutamyl transferase (268 U/L [5-61]) and alkaline phosphatase (346 U/L [35-104]).

Pending staging, a percutaneous transhepatic colangiography (PTC) to decompress the bile duct was performed. A $10 \mathrm{~F}$ plastic stent with internal and external drainage was placed.

The patient was then proposed for palliative treatment and referred to our Gastroenterology Department for definitive internal drainage. Although a percutaneous SEMS would be technically simpler as the patient already had a percutaneous access, this was not logistically possible due to temporary absence of interventional radiologists in the following weeks.

Using the rendez-vous technique, a single balloon enteroscopy assisted ERCP (SBE-ERCP) was planned.

Previously, the external drainage was removed and a guidewire $(0.035$ " with $150 \mathrm{~cm}$, Amplatz Super Stiff J-tip, Cordis) was left in place.

The single balloon enteroscope (Olympus SIF-Q180, Japan) was used. The enteroscope is $200 \mathrm{~cm}$ long and has a $2.8 \mathrm{~mm}$ working channel. The overtube (ST-SB1, Olympus, Japan) is a $140 \mathrm{~cm}$ long silicone overtube with an $11 \mathrm{~mm}$ internal diameter, with a pressure controlled balloon at the distal end. The enteroscope was advanced through the afferent limb to the papila. A 4 Fr catheter $(0.038$ " with $65 \mathrm{~cm}$, Cordis) was then placed over the wire and the guidewire removed. A $500 \mathrm{~cm} \mathrm{0.0035'} \mathrm{guidewire} \mathrm{(Endoflex} \mathrm{21535500,}$ Germany) was placed percutaneously through the catheter and grasped with a polypectomy snare. The enteroscope was pulled en bloc outside with the polypectomy snare grasping the guidewire, leaving the overtube and percutaneous catheter in place. This is technically easier and less damaging to the enteroscope, than pulling the guidewire through a narrow $2.8 \mathrm{~mm}$ and $200 \mathrm{~cm}$ long biopsy channel.

A $280 \mathrm{~cm}$ sphincterotome (Endoflex OE1042230DL-280, Germany) was placed through the enteroscope working channel and the guidewire was inserted retrogradely through the sphincterotome's tip. The enteroscope was then advanced with the sphincterotome in place over the wire, through the overtube, to the papilla, and deep biliary cannulation was performed. The cholangiogram revealed stenosis of the distal 2/3 of the bile duct (Fig. 1). Limited sphincterotomy was performed.

As the use of a standard self-expanding metallic stent through the enteroscope's $2.8 \mathrm{~mm}$ working channel is impossible, a novel technique was used. The overtube was advanced and placed in front of the papilla. The balloon at the tip of the overtube was inflated and a pull maneuver was performed. Contrast was injected in the duodenum to create a submucosal bleb adjacent to the papilla and the enteroscope was removed, leaving the overtube and guidewire in place. A standard biliary SEMS (Hanarostent NNN 10x60 mm, M.I. Tech Co, Korea) was then advanced under fluoroscopic control, over the wire and through the over-

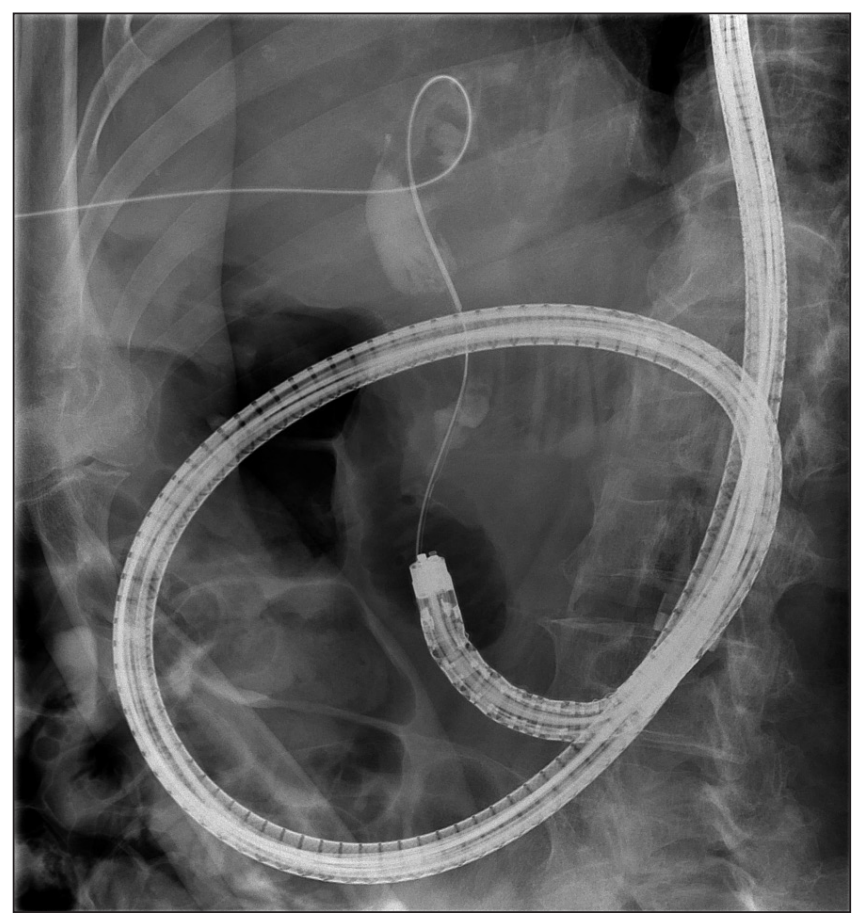

Fig. 1. Cholangiogram showing distal common bile duct stenosis.

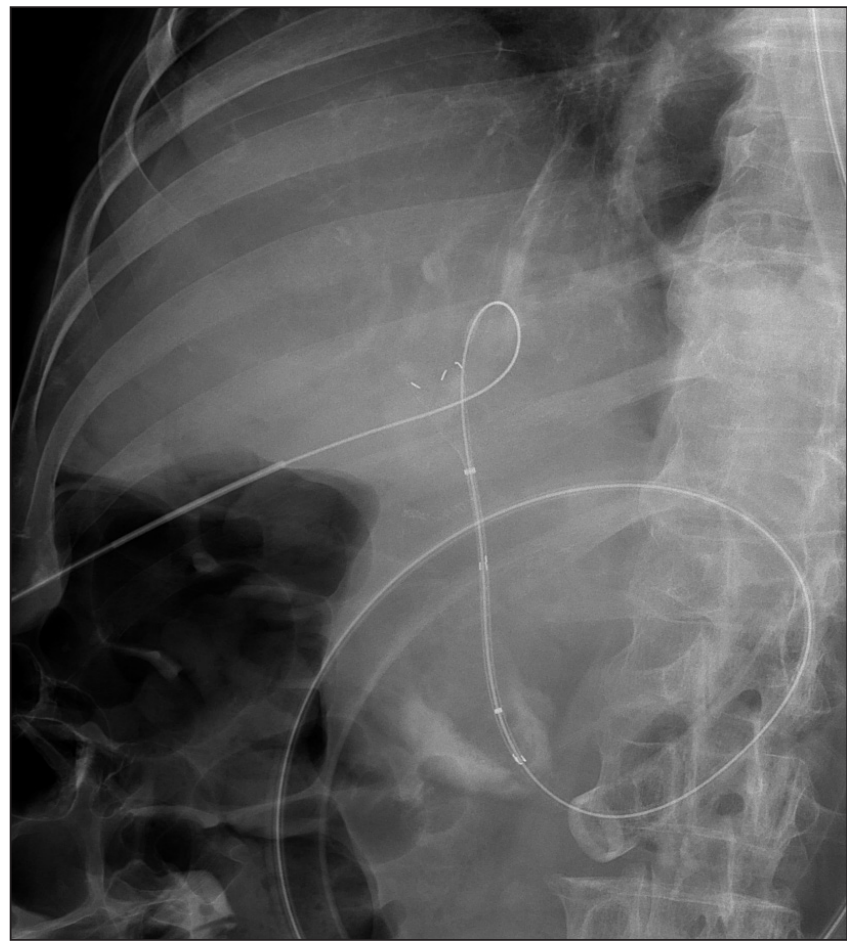

Fig. 2. A standard biliary SEMS is placed over the wire, through the overtube using fluoroscopic control. The submucosal contrast bleb near the papilla is used as a reference. 
tube, to the bile duct (Fig. 2). The SEMS was positioned using the contrast bleb near the papilla as a reference. After deployment, inadequate expansion of the proximal end of the stent was apparent (Fig. 3). A second SEMS (Hanarostent NNN 10 x 100 mm, M.I. Tech Co, Korea) was then placed proximally using the same technique and complete palliation was confirmed fluoroscopically (Fig. 4).

Although a temporary standard 7 Fr plastic stent could be easily placed through the $2.8 \mathrm{~mm}$ working channel, a biliary SEMS was preferred to obtain definitive palliation.

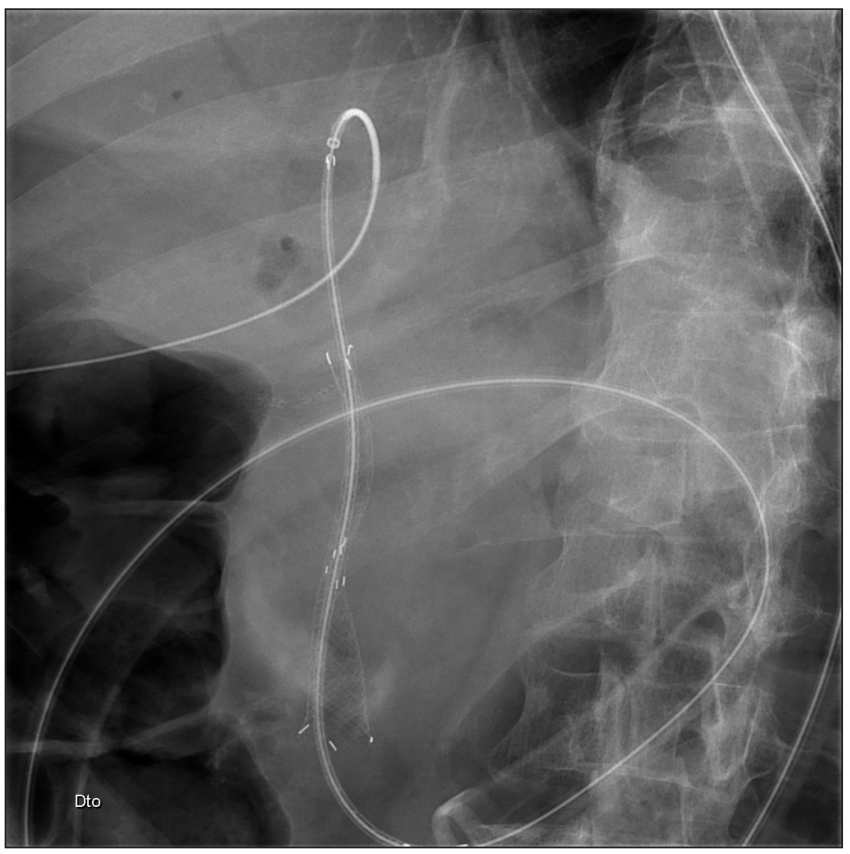

Fig. 3. Placement of a second biliary SEMS using the same technique.

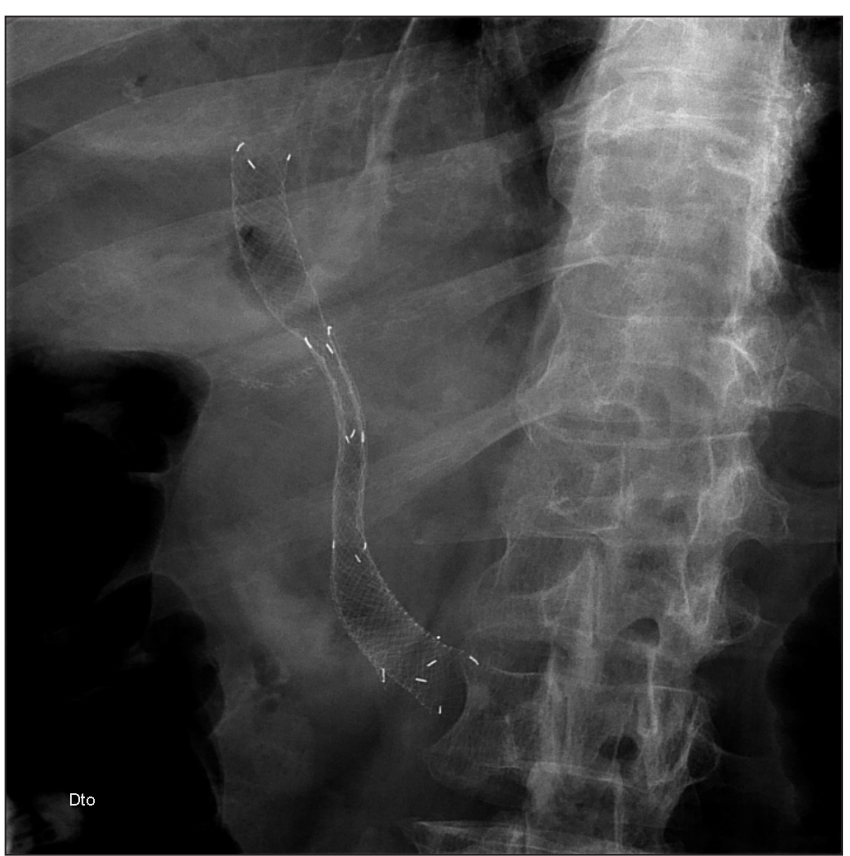

Fig. 4. Final result showing complete palliation by the 2 biliary SEMS.
The procedure was uneventful.

Mild asymptomatic hyperamilasemia (273 U/L [13-53]) was observed the next day. The patient was discharged 6 days later with normal amylase, mild hyperbilirubinemia (total bilirubin $1.8 \mathrm{mg} / \mathrm{dL}$ [0.1-1.1], conjugated bilirubin $1.41 \mathrm{mg} / \mathrm{dL}$ [0.1-0.3]), and lower gamma-glutamyl transferase (74 U/L [5-61]) and alkaline phosphatase (228 U/L [35-104]).

The patient is currently in the $6^{\text {th }}$ month of follow-up, with no recurrence of biliary obstruction, jaundice or hyperbilirubinemia (total bilirubin $0.42 \mathrm{mg} / \mathrm{dL}$ [0.1-1.1]).

\section{DISCUSSION}

ERCP is the gold standard for the diagnosis and therapy of bile duct pathology (7). With the development of DAE-ERCP, access to the pancreatic and biliary duct systems in patients with surgically altered anatomies is now possible, although technically challenging, difficult and time-consuming. Therapeutic interventions such as sphincterotomy, balloon dilation, stone extraction and plastic stent placement have all been demonstrated to be technically feasible and safe. The main difficulties are related to the diameter and length of the working channel of the enteroscope that renders standard ERCP accessories impossible to use. Standard self expanding metallic stents can't be introduced in the channel of current enteroscopes and dedicated SEMS with longer introducer length and smaller diameter have not yet been developed.

A new technique for SEMS deployment using SBE-ER$\mathrm{CP}$ in patients with surgically altered anatomy that renders standard ERCP impossible has been developed and proved to be feasible and safe. A previous report of a SEMS placed with the aid of DAE-ERCP has been published (8), but in that case report the stent was placed percutaneously (not endoscopically) with the aid of DAE-ERCP. Another report of SEMS placement with DBE-ERCP in a patient with Roux-en-Y reconstruction was published (9) but the $152 \mathrm{~cm}$ short DBE was used with the standard through the scope technique. Short DBE scopes or thinner colonoscopes to use with the overtube are however not widely available. In another report (10), balloon enteroscopy was used to create a percutaneous endoscopic gastrostomy (PEG) in a patient with a gastric bypass surgery. A transgastric SEMS was used to protect the freshly created PEG site. Then, using transgastric access through the PEG, a standard duodenoscope was used to perform ERCP. Although not applicable to our case report, this technique could be used to place biliary SEMS in gastric bypass patients using the standard through-the-scope technique.

ERCP is usually preferred over PTC as it is a less invasive procedure. Patients with surgically altered anatomy, which were previously referred for percutaneous drainage, are increasingly being managed with DAE-ERCP. The percutaneous approach to place biliary SEMS is technically 
simple in this setting and has been the preferred technique for that purpose. However it is not always effective (8) and available and this report demonstrates that DAE-ERCP can also be used for this indication.

This is the first report of a SEMS placed in the biliary duct through the papilla, using DAE-ERCP with a standard enteroscope, proving that SEMS can also be placed with this technique and that available biliary SEMS can be used for that purpose. Although the procedure was performed after a PTC, using the rendez-vous approach, the same "through-the-overtube and over-the-wire" technique can theoretically be used without a previous PTC. This technique can also be used with DBE-ERCP or SE-ERCP and with other standard biliary SEMS.

\section{REFERENCES}

1. Pohl J, Delvaux M, Ell C, Gay G, May A, Mulder CJ, et al. European Society of Gastrointestinal Endoscopy (ESGE) Guidelines: Flexible enteroscopy for diagnosis and treatment of small-bowel diseases. Endoscopy 2008;40:609-18.
2. Emmett DS, Mallat DB. Double-balloon ERCP in patients who have undergone Roux-en-Y surgery: A case series. Gastrointest Endosc 2007;66:1038-41.

3. Mönkemüller K, Fry LC, Bellutti M, Neumann H, Malfertheiner P. ERCP using single-balloon instead of double-balloon enteroscopy in patients with Roux-en-Y anastomosis. Endoscopy 2008; 40(Supl. 2): E19-20.

4. Wang AY, Sauer BG, Behm BW, et al. Single-balloon enteroscopy effectively enables diagnostic and therapeutic retrograde cholangiography in patients with surgically altered anatomy. Gastrointest Endosc 2010; 71:641-9

5. Kogure H, Watabe H, Yamada A. Spiral enteroscopy for therapeutic ERCP in patients with surgically altered anatomy: Actual technique and review of the literature. J Hepatobiliary Pancreat Sci 2011;18:375-9.

6. Moreels TG. Altered anatomy: Enteroscopy and ERCP procedure. Best Pract Res Clin Gastroenterol 2012;26:347-57.

7. O'Mahony S, Lintott DJ, Axon A. Endoscopic retrograde cholangiopancreatography. Semin Laparosc Surg 1995;2:93-101.

8. Koornstra JJ, Alkefaji H. Self-expandable metal stent placement combining double balloon endoscopy with a percutaneous approach in a Roux-en-Y hepaticojejunostomy. J Gastrointestin Liver Dis 2009;18:375-7.

9. Tsutsumi K, Kato H, Tomoda T, Matsumoto K, Sakakihara I, Yamamoto $\mathrm{N}$, et al. Partial stent-in-stent placement of biliary metallic stents using a short double-balloon enteroscopy. World J Gastroenterol 2012;18:6674-6

10. Baron TH, Song LM, Ferreira LE, Smyrk TC. Novel approach to therapeutic ERCP after long-limb Roux-en-Y gastric bypass surgery using transgastric self-expandable metal stents: Experimental outcomes and first human case study. Gastrointest Endosc 2012;75:1258-63. 